\title{
Constraints on interstellar dust models from extinction and spectro-polarimetry
}

\author{
R. Siebenmorgen \\ European Southern Observatory, Karl-Schwarzschild-Str. 2, D-85748 Garching b. München, Germany, \\ Ralf.Siebenmorgen@eso.org \\ N.V. Voshchinnikov \\ Sobolev Astronomical Institute, \\ St. Petersburg University, Universitetskii prosp. 28, St. Petersburg, 198504 Russia \\ S. Bagnulo \\ Armagh Observator and Planetariumy, College Hill, Armagh BT61 9DG, UK \\ N.L.J. Cox \\ Anton Pannekoek Institute for Astronomy, University of Amsterdam, NL-1090 GE Amsterdam, The \\ Netherlands.
}

\begin{abstract}
We present polarisation spectra of seven stars in the lines-of-sight towards the Sco OB1 association. Our spectra were obtained within the framework of the Large Interstellar Polarization Survey carried out with the FORS instrument of the ESO VLT. We have modelled the wavelength-dependence of extinction and linear polarisation with a dust model for the diffuse interstellar medium which consists of a mixture of particles with size ranging from the molecular domain of $0.5 \mathrm{~nm}$ up to $350 \mathrm{~nm}$. We have included stochastically heated small dust grains with radii between 0.5 and $6 \mathrm{~nm}$ made of graphite and silicate, as well as polycyclic aromatic hydrocarbon molecules (PAHs), and we have assumed that larger particles are prolate spheroids made of amorphous carbon and silicate. Overall, a dust model with eight free parameters best reproduces the observations. Reducing the number of free parameters leads to results that are inconsistent with cosmic abundance constraints. We found that aligned silicates are the dominant contributor to the observed polarisation, and that the polarisation spectra are best-fit by a lower limit of the equivolume sphere radius of aligned grains of $70-$
\end{abstract}

Preprint submitted to Journal of $\mathrm{BT}_{E} X$ Templates

July 14, 2021 
$200 \mathrm{~nm}$.

Keywords: dust, extinction, polarisation, interstellar medium

\section{Introduction}

Our understanding of many astrophysical processes, ranging from galaxy evolution to stellar and planetary formation, depends on our knowledge of the cosmic dust (e.g. Draine [19], Asano et al. [5]). Dust and molecular gas co-exist in interstellar clouds, and the properties of both components are mutually dependent through various chemical reactions and physical (photon) processes.

Interstellar dust grains absorb, scatter, and polarise the background radiation. The observed wavelength dependencies of extinction, emission and polarisation of the radiation coming from background sources may allow us to characterise the dust of the diffuse interstellar medium (ISM) (Désert et al. [15], Siebenmorgen, \& Krügel [67], Kim et al. [46], Dwek et al. [26], Weingartner \& Draine [80], Draine \& Li [23], Draine \& Fraisse [20], Jones et al. [45], Köhler et al. [47], Voshchinnikov [73], Siebenmorgen et al. [66], Voshchinnikov et al. [76]).

The absolute extinction is often represented as a function $A\left(\lambda^{-1}\right)$ normalised by the extinction value in the visible band $A_{V}$. This extinction curve $A\left(\lambda^{-1}\right)$ has been characterised for hundreds of sight lines (Fitzpatrick \& Massa [31, 32], Calzetti et al. [10], Papaj \& Krelowski [61], Fitzpatrick \& Massa [33], Valencic et al. [72], Gordon et al. [38]). It varies with wavelength and approaches zero at longer wavelengths. In the optical, the extinction curves tend to follow a relation that depends on the total-toselective extinction $R_{V}=A_{V} / E_{B-V}$ (Cardelli et al. [11], see also Fitzpatrick \& Massa [33]), where $E_{B-V}=(B-V)-(B-V)_{0}$ is the colour excess given by the observed magnitude difference at $B$ and $V$ of the star and of identical unreddened star (Johnson [43], Bless \& Savage [8], Massa et al [55], Massa [54]). Flat extinction curves with large $R_{V}$ values are measured towards denser regions. The parameter $R_{V}$ is also a rough indicator of grain size: sight lines of low $R_{V}$ values are thought to be characterised by smaller grains than sight lines with higher $R_{V}$ values.

Extinction curves may be modified by scattering of photons from dust clouds in 
or out of the line of sight. Scicluna \& Siebenmorgen [64] have shown that the observed extinction is not significantly modified by scattering from dust clouds at distance smaller than $1 \mathrm{kpc}$. For such nearby stars, the extinction curves provide crucial information on the composition and size distribution of the interstellar dust.

Also polarisation spectra constrain sizes, shape and composition of the dust grains. Asymmetrical dust grains are most likely aligned with the magnetic field and polarise the light (Andersson et al. [2]). So far, the majority of polarisation measurements are obtained in optical $(U B V R)$ and near infrared $(I J H K)$ broadband filters (Whittet [81]). The observed polarisation spectra generally have a quasi-parabolic shape (Serkowski et al. [65]). The position of the polarisation maximum and the width of the polarisation spectrum varies from star to star, and this diversity is probably due to different local conditions affecting the alignment efficiencies and the grain sizes (Voshchinnikov \& Hiroshita [79]). Insight into the shape and size distribution and composition of interstellar grains may come from detailed physical models (Draine \& Fraisse [20], Voshchinnikov [73], Siebenmorgen et al. [66], Voshchinnikov et al. [76]).

We have started a Large Interstellar Polarisation Survey (LIPS; PI: N.J.L. Cox) with the aim of determining the chemical composition and size distribution of interstellar dust in numerous sight-lines. In this paper we present spectro-polarimetry of seven early-type stars obtained with the FORS2 instrument (Appenzeller et al. [4]) of the ESO VLT. Spectra cover the wavelength range $365-920 \mathrm{~nm}$ and have a resolution of about 880. For our sample of stars, extinction curve measurements are available by Valencic et al. [72]. The seven stars are located towards sight lines of the ScorpiusCentaurus Association (called Sco OB1).

This paper is organised as follows. In Sect. 2 we present our new data. In Sect. 3 we present a dust model and a procedure for fitting the extinction and polarisation curve of an object simultaneously. Sect. 4 presents the result of our modeling efforts. In Sect.5 we give a summary of our main results. 


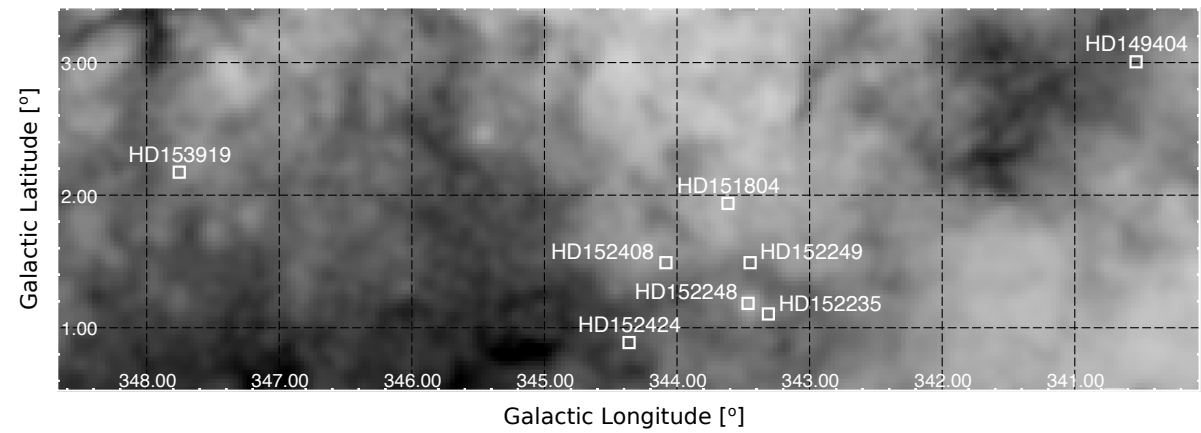

Figure 1: Observed stars towards Sco OB1 displayed on top of an extinction map produced from DSS (Dobashi et al. [16]).

\section{Observations}

From the original LIPS target list we selected a small sub-sample comprising seven close-by lines-of-sight towards the Sco OB1 region, including two possible association members, HD 151804, HD 152235 that are supergiants stars.

In Fig 1 we show the target location in galactic coordinates overlaid to an extinction map produced from DSS (Dobashi et al. [16]). The distance for HD 153919 is 1.7 kpc (Ankay et al. [3]) while the other stars (HD 151804, HD 152235, HD 152248, HD 152408), are confirmed Hipparcos members of Sco OB1 which is at an estimated distance of $2 \mathrm{kpc}$. The stars HD 152235, HD 152248, HD 152249 and HD 152408, are close to each other in the sky, and have low polarisation efficiency of $p_{\max } / E(B-V) \sim$ $1-2$. More detailed target information is given in Table 1 .

\subsection{Extinction}

Extinction curves are usually measured with ground-based facilities in the near IR, optical and UV photometric bands, and, at shorter UV wavelengths, by the IUE $\left(3.3 \mu \mathrm{m}^{-1}<\lambda^{-1}<8.6 \mu \mathrm{m}^{-1}\right)$ and FUSE $\left(3.3 \mu \mathrm{m}^{-1}<\lambda^{-1}<11 \mu \mathrm{m}^{-1}\right)$ satellites, which provide spectroscopy at a resolution of $\sim 0.5 \mathrm{~nm}$. Extinction data are often fit by a third order polynomial and a Drude profile to account for the $217 \mathrm{~nm}$ extinction bump (Fitzpatrick \& Massa [32, 33]). Extinction curves of our sample stars are provided by Valencic et al. [72] using IUE data, while FUSE observations at $\lambda^{-1}<8.6 \mu \mathrm{m}^{-1}$ are 
Table 1: Observing sample.

\begin{tabular}{lllllll}
\hline \hline Target & Sp.Type & $l$ & $b$ & $\mathrm{~V}$ & $\mathrm{E}(\mathrm{B}-\mathrm{V})$ & $\mathrm{R}(\mathrm{V})$ \\
$(1)$ & $(2)$ & $(3)$ & $(4)$ & $(5)$ & $(6)$ & $(7)$ \\
\hline HD 149404 & O8.5Iab(f)p & 340.5375 & +03.0058 & 5.87 & $0.62 \pm 0.06$ & $3.53 \pm 0.38$ \\
HD 151804 & O8Iaf & 343.6156 & +01.9378 & 5.29 & $0.30 \pm 0.03$ & $4.33 \pm 0.30$ \\
HD 152235 & B0.5Ia & 343.3111 & +01.1041 & 6.38 & $0.71 \pm 0.06$ & $3.13 \pm 0.25$ \\
HD 152248 & O7Iabf+O7Ib & 343.4644 & +01.1839 & 6.15 & $0.41 \pm 0.04$ & $3.68 \pm 0.26$ \\
HD 152249 & OC9Iab & 343.4488 & +01.1649 & 6.65 & $0.46 \pm 0.10$ & $3.54 \pm 0.45$ \\
HD 152408 & O8Iape & 344.0836 & +01.4909 & 5.92 & $0.42 \pm 0.05$ & $4.17 \pm 0.33$ \\
HD 153919 & O6Iafcp & 347.7544 & +02.1735 & 6.78 & $0.51 \pm 0.04$ & $3.87 \pm 0.22$ \\
\hline
\end{tabular}

NOTES - Target name (1), Spectral type of star (2), galactic longitude $l$ (3) and latitude $b$ (4), $V$ from Simbad (5), $E(B-V)(6)$ and $R(V)$ (7) by Valencic et al. [72].

not available for our stars. Following Gordon et al. [38], we decreased the value of the parameter describing the far UV rise as given by Valencic et al. [72] by $\sim 8 \%$; other fit parameters determined with IUE data, or those obtained using the combined IUE and FUSE data sets, are broadly consistent (Gordon et al. [38]). In order to fit the extinction data in the $U B V J H K$ bands we have used the $R_{V}$ parametrisation by Fitzpatrick [30].

Extinction curves of the ISM that are derived from observations of supergiants might be affected by systematic errors that are larger than for main sequence stars. For supergiants it is difficult to disentangle the ISM extinction from contribution by circumstellar dust, and it is difficult in finding an unreddened supergiant with spectral class. The extinction curves of our sample stars have typical uncertainties of $10 \%$ in the optical/UV with somewhat larger uncertainties in the near infrared.

\subsection{Polarimetry with FORS2}

Linear spectro-polarimetric observations were obtained with the FORS2 instrument of the ESO VLT (Appenzeller et al. [4]) within the context of the Large Interstellar 
Table 2: FORS/VLT spectro-polarimetric observation log. Columns 2 and 3 refer to the date and time of the observations, col. 4 is the exposure time in secs, and col. 5 provides the $\mathrm{S} / \mathrm{N}$ per $\AA$ at peak level.

\begin{tabular}{ccccc}
\hline \hline Star & $\begin{array}{c}\text { Date } \\
\text { (yyyy-mm-dd })\end{array}$ & $\begin{array}{c}\text { UT } \\
(\mathrm{hh}: \mathrm{mm})\end{array}$ & $\begin{array}{c}\text { EXP } \\
(\mathrm{s})\end{array}$ & $\begin{array}{c}\mathrm{S} / \mathrm{N} \\
(\text { per } \AA)\end{array}$ \\
$(1)$ & $(2)$ & $(3)$ & $(4)$ & $(5)$ \\
\hline HD 149404 & $2015-05-12$ & $05: 40$ & 1.6 & 80 \\
HD 151804 & $2015-05-24$ & $03: 41$ & 1.6 & 75 \\
HD 152335 & $2015-05-24$ & $04: 01$ & 2.0 & 70 \\
HD 152248 & $2015-05-24$ & $04: 18$ & 2.0 & 75 \\
HD 152249 & $2015-05-24$ & $04: 34$ & 4.0 & 80 \\
HD 152408 & $2015-05-24$ & $04: 49$ & 1.6 & 80 \\
HD 153919 & $2015-04-12$ & $09: 01$ & 4.0 & 80 \\
\hline
\end{tabular}

Polarisation Survey (LIPS, PI: N.J.L. Cox). All observations were obtained with grism $300 \mathrm{~V}$, which covers the spectral range $365-920 \mathrm{~nm}$, using the so-called beam-swapping technique recommended in the FORS user manual, and described in detail, e.g., by Bagnulo et al. [7]. With a $0.5^{\prime \prime}$ slit width, our observations have a nominal spectral resolution $\lambda / \Delta \lambda \sim 880$. Data were reduced following the guidelines by Bagnulo et al. [7]. Note that null profiles could not be determined since observations were obtained with four exposures only (at the following four positions of the retarder wave-plate: $0^{\circ}, 22.5^{\circ}, 45^{\circ}, 67.5^{\circ}$ ). To improve the $\mathrm{S} / \mathrm{N}$ ratio, we decided to rebin our spectra, by degrading the spectral resolution to $\lambda / \Delta \lambda \sim 50$. In the rebinning process, outliers (e.g., due to cosmic-rays) were rejected with a $3 \sigma$ clipping algorithm; after outlier rejection, we calculated the weighted mean of the pixel values, and estimating $1 \sigma$ error from the variance of the polarisation of the original bin. In Table 2 we specify the observing date and time, the on source exposure time, and the mean signal-to-noise per $\AA$. 


\section{Model}

In this section we describe our technique for simultaneous modelling of UV to IR dust extinction and linear polarisation curves observed towards the diffuse ISM.

\subsection{Dust populations}

Data cannot be fit by assuming that all dust particle have the same size, but we need to introduce a dust grain size distribution. In particular we use a power law size distribution $n(r) \propto r^{-q}$ as introduced by Mathis et al. [56]. Particle sizes range from the molecular $\left(r_{-} \sim 0.5 \mathrm{~nm}\right)$ to the sub-micrometer domain.

Interstellar polarisation cannot be explained by spherical particles made of optically isotropic material, but we need to assume that some dust particles are of non-spherical shape, and partly aligned. Therefore we have considered spheroidal dust particles. In the following we will denote with $a$ and $b$ their major and minor semi-axis, respectively. There exist two types of spheroids: oblates (or 'disks') and prolates (or 'needles'). Oblate spheroids are obtained by a rotation of an ellipse around the minor $(b)$ axis and prolates are obtained by a rotation of an ellipse around the major $(a)$ axis. The sizes of spheroids can be compared if we use the sphere of radius $r$ which volume equal to that of spheroid: $r^{3}=a b^{2}$ for prolate spheroids and $r^{3}=a^{2} b$ for oblate ones. We found that prolate particles provide better fits to the polarisation spectra than oblate particles.

Voshchinnikov [73], Das et al. [14], Siebenmorgen et al. [66] computed extinction $C_{\text {ext }}(v)$, scattering $C_{\text {sca }}(v)$, and linear polarisation $C_{\mathrm{p}}(v)$ cross sections and corresponding efficiency factors $Q=C / \pi r^{2}$ using the separation of variables method presented by Voshchinnikov \& Farafonov [77]. Subsequently, cross sections were averaged over size distribution and rotations.

In our dust model we include large homogeneous spheroids that are made up of silicate and amorphous carbon, and a population of small graphite and small silicates, as well as a PAH component with $60 \mathrm{H}$ atoms and $150 \mathrm{C}$ atoms, with cross sections $C_{\mathrm{PAH}}(v)$ as given by Siebenmorgen et al. [66]. Dust cross-sections of prolate particles are computed for 100 radius values between 6 and $800 \mathrm{~nm}$. Small grains have radius $r$ such that $0.5 \mathrm{~nm} \leq r \leq 6 \mathrm{~nm}$. Draine \& Hensley [22] pointed out that the rapid 
fall-off in starlight polarisation in the far UV suggests that grains in this size range are either nearly spherical, or do not contribute to the far UV extinction. In our model they contribute to the far UV extinction and do not contribute to the polarisation curve. Therefore we assume that grains below $6 \mathrm{~nm}$ have spherical shape. We apply for large silicates and carbon particles different upper size limits $r_{+}^{\mathrm{Si}}$ and $r_{+}^{\mathrm{aC}}$.

For graphite, the dielectric function is anisotropic and average extinction efficiencies are computed by setting $Q=2 Q\left(\epsilon^{\perp}\right) / 3+Q\left(\epsilon^{\|}\right) / 3$, where $\epsilon^{\perp}, \epsilon^{\|}$are dielectric constants for two orientations of the $\vec{E}$ vector relative to the basal plane of graphite (Draine \& Malhotra [24]). Efficiencies in both directions are computed via Mie theory. Optical constants for large silicates and graphite are given by Draine [17]. Hydrogen-free amorphous carbon becomes hydrogenated, when exposed to atomic hydrogen (Furton \& Witt [34]). We employ the ACH2 mixture by Zubko et al. [85]. This are hydrogenated amorphous carbon particles and semiconductors. Densities of these grains depend on their sp2/sp3 ratio and hydrogen content, and range from $1.2-2.2\left(\mathrm{~g} / \mathrm{cm}^{3}\right)$ (Robertson [63], Casiraghi et al. [12]). Furton et al [35] characterized the interstellar absorption feature at $3.4 \mu \mathrm{m}$ by such grains and estimate a mass density of $1.5 \mathrm{~g} / \mathrm{cm}^{3}$ for them. We take a bulk density $\rho_{C} \sim 1.6 \mathrm{~g} \mathrm{~cm}^{-3}$ for carbon and $\rho_{\mathrm{Si}} \sim 3.5 \mathrm{~g} \mathrm{~cm}^{-3}$ for silicates. We also computed dust cross sections with optical constants provided by Jones [44] and Jones et al. [45]. We find that in the optical/UV the dust cross sections are similar for either set of constants, whereas differences in the cross sections become strong at wavelengths $\geq 1 \mu \mathrm{m}$. In summary, four different dust populations are considered and in the following labelled as large silicates ( $\mathrm{Si})$, large amorphous carbon $(\mathrm{aC})$, small silicates (sSi), small graphite (gr) and PAH.

We fit the extinction curve, or equivalently the observed optical depth profile, for each star of our sample by the extinction cross section of the dust model. The total dust extinction cross section $K_{\text {ext }}(v)$ is given in $\mathrm{cm}^{2} \mathrm{~g}^{-1}$. It is wavelength dependent and given as the sum of the absorption and scattering coefficient of the dust materials. For each dust component there is a specific (relative) dust abundance. It is denoted by $\Upsilon$ together with a subscript for each dust population $\mathrm{Si}, \mathrm{aC}, \mathrm{sSi}, \mathrm{gr}, \mathrm{PAH}$. The specific dust abundance avoids introducing systematic errors of present estimates of the gas phase depletion of an element (Draine [18], Dwek [27], Gerin et al. [36], Nieva \& 
Przybilla [60], Jenkins [42], Parvathi et al. [62], Voshchinnikov \& Henning [78]). Formulas for computing $K_{\text {ext }}$ and $\Upsilon$ are given in Siebenmorgen et al. [66].

\subsection{Linear polarisation}

The extinction cross section of non-spherical particles depends on their orientation with respect to the electric field of the incident light. Unpolarised stellar light that passes a cloud of moderate extinction by aligned grains becomes linearly polarised. In the spectral region from the far UV to the near IR, the observed polarisation curves may be fit by the empirical formula given by Serkowski et al. [65]

$$
p(\lambda)=p_{\max } \exp \left[-k_{\mathrm{p}} \ln ^{2}\left(\frac{\lambda_{\max }}{\lambda}\right)\right] .
$$

Equation (1) includes three parameters: the maximum polarisation $p_{\max }$, the wavelength $\lambda_{\max }$ at $p_{\max }$, and the width of the spectrum $k_{\mathrm{p}}$. Voshchinnikov et al. [76] studied observations of 160 sight lines of mildly reddened stars. They report that the observed linear polarisation is of a few percent, $p / E_{B-V} \leq 9 \% / \mathrm{mag}$ and its maximum $p_{\max }$ is always $<10 \%$. The width of the polarisation curve is $0.5 \leq k_{\mathrm{p}} \leq 1.5$, and $\lambda_{\max }$ is often observed close to the $V$ band. It varies between $0.35-0.8 \mu \mathrm{m}$ and exceptionally up to $\sim 1 \mu \mathrm{m}$ in dark clouds (Goodman et al. [37]).

Observed values of the Serkowski parameters can be modeled assuming aligned and homogeneous spheroidal sub-micrometer sized dust particles made of silicates and carbon. One simplifies the alignment process assuming picket fence (Krügel [48], Draine \& Fraisse [20]), the radiative torque (RAT, Lazarian [51], Andersson et al. [2]) or the imperfect Davies Greenstein (IDG) alignment (Voshchinnikov [73]). For the diffuse medium, the IDG function is often applied. It is analytic and depends on particle size, dust precession angle, the dust-to-gas temperature ratio $T_{\mathrm{d}} / T_{\mathrm{g}}$, and an alignment efficiency parameter $\delta_{0}$. The maximum polarisation depends strongly on $\delta_{0}$ and on the magnetic field orientation $\Omega$, which is the angle between $B$ and the line of sight. The two quantities are difficult to be disentangled from each other. Fortunately, they have little influence on the normalised polarisation curve, which is parameterised by $\lambda_{\max }$ and $k_{\mathrm{p}}$. 
The influence of dust parameters on polarisation was studied by Das et al. [14], Voshchinnikov \& Hiroshita [79], Siebenmorgen et al. [66], Voshchinnikov et al. [76], who considered a dust size distribution with the equivolume sphere radius ranging from $r_{-}^{\mathrm{pol}}$ up to $r^{+}$. They found that oblate spheroids produce narrow polarisation curves often outside the observed values of $\lambda_{\max }$ and $k_{\mathrm{p}}$, and that they are more efficient polarisers than prolates (hence they may explain larger values of $p_{\max }$ ). The aspect ratio $a / b$ of the spheroids has no strong impact on the Serkowski curve. Adopting prolate grains generally leads to better fits to polarisation data. As the ratio $a / b$ between the semiaxis of the prolates increases, the Serkowski curve widens and the $\lambda_{\max }$ value decreases. The exponent $q$ of the size distribution of prolates does not influence the polarisation strongly. The model parameters $r_{+}$and more so $r_{-}^{\mathrm{pol}}$ are those with the major impact on the polarisation curve.

In this work, we model the observed linear polarisation using

$$
\frac{p(v)}{A_{V}}=\frac{K_{\mathrm{p}}(v)}{K_{\mathrm{ext}, V}} .
$$

where $K_{\mathrm{p}}(v)$ is the linear polarisation cross section of the dust (Voshchinnikov [73], Siebenmorgen et al. [66]).

\subsection{Fitting procedure}

We computed the various dust cross-sections in the spectral range from $70 \mathrm{~nm}$ up to $2 \mathrm{~mm}$ and for grain radius between $0.5 \mathrm{~nm}$ and $800 \mathrm{~nm}$. We defined a grid size by setting $r_{i+1}=1.05 r_{i}$, and we linearly interpolated the modelling results for the size values intermediate between two consecutive grid points. We considered a different upper grain radius for silicates and carbon. The other free parameters of our models are the exponent of the dust size distribution and the specific dust abundances.

We modelled the shape of the extinction and polarisation curves and not their absolute properties, therefore it was sufficient to specify for the $n=5$ dust populations only $n-1$ specific dust abundances $\Upsilon$. The abundances could be scaled up or down by an arbitrary factor without any change in the predicted extinction or polarisation curves. We experimented with a normalisation of the specific dust abundances $\Upsilon$ that allows a direct comparison and consistency check with absolute abundance constraints. 
We set $\Upsilon_{\mathrm{Si}}=15 \mathrm{ppm}$ in large silicate grains, which equals the lower limit derived by Voshchinnikov \& Henning [78].

We modeled the shape of the polarisation curve but not its absolute value $p_{\max }$, which allows to keep the IDG alignment parameters and the field orientation constant. We use $T_{\mathrm{d}} / T_{\mathrm{g}}=0.1, \delta_{0}=10 \mu \mathrm{m}$, and $\Omega=90^{\circ}$ (for a study of the influence of these parameters on the polarisation, see Voshchinnikov et al. [76]). We considered prolate shaped large silicate grains with axial ratio $a / b=2$. We made the further simplifying assumptions that the largest radius of aligned silicates and the exponent of the size distribution is the same as derived by fitting the extinction curve, so that for the fit to the polarisation curves we were left with one free parameter $r_{-}^{\mathrm{pol}}$.

We applied the dust model and fit the extinction and polarisation curves with eight free parameters: $q, \Upsilon_{\mathrm{aC}}, \Upsilon_{\mathrm{gr}}, \Upsilon_{\mathrm{PAH}}, \Upsilon_{\mathrm{sSi}}, r_{-}^{\mathrm{pol}}, r_{+}^{\mathrm{Si}}$, and $r_{+}^{\mathrm{aC}}$. Best-fit parameters and their $1 \sigma$ errors are computed by a least-square technique utilizing the Levenberg-Marquardt algorithm as implemented in MPFIT 1] (Markwardt [53], More [57], More \& Wright [58]). The algorithm is able to find the local minima, and to identify the global minimum we need to apply it starting from many different initial values. For each particle radius $100 \mathrm{~nm}<r_{+}^{\mathrm{aC}}<800 \mathrm{~nm}$ we computed the reduced $\chi^{2}$ of the fit to the extinction $\chi_{\mathrm{e}}^{2}(r)$ and polarisation $\chi_{\mathrm{p}}^{2}(r)$ curve. The model that simultaneously fits both curves best was selected from the minimum $\chi$ condition and where each of the eight fitting parameters is given the same weight. We found that in our model $\chi^{2}(r)$ was generally well behaved showing a (nearly) parabolic shape and a global minimum.

\section{Results}

In this Sect. we present our best-fits to the extinction and polarisation spectra of the seven targets of Sect. 2 obtained by applying the dust model and fitting procedure described in Sect. 3 . We will call the model with eight free parameters 'p8'. Figures 2 - 8 show the data and best-fits for each target, and are organised as follows. In the upper panel we display the extinction data $\tau\left(\lambda^{-1}\right) / \tau_{V}$ of the target with a white dashed

\footnotetext{
${ }^{1}$ http://purl.com/net/mpfit
} 
Table 3: Fit parameters of the dust models.

\begin{tabular}{|c|c|c|c|c|c|c|c|c|}
\hline Target & $\begin{array}{c}{[\mathrm{Si}]^{\mathrm{tot}} /[\mathrm{H}]} \\
(\mathrm{ppm}) \\
(1)\end{array}$ & $\begin{array}{r}{[\mathrm{C}]^{\mathrm{tot}} /[\mathrm{H}]} \\
(\mathrm{ppm}) \\
(2)\end{array}$ & $\begin{array}{r}{[\mathrm{C}]^{\mathrm{gr}} /[\mathrm{H}]} \\
(\mathrm{ppm}) \\
(3)\end{array}$ & $\begin{array}{c}{[\mathrm{C}]^{\mathrm{PAH}} /[\mathrm{H}]} \\
(\mathrm{ppm}) \\
(4)\end{array}$ & (5) & $\begin{array}{l}r_{-}^{\mathrm{pol}} \\
(\mathrm{nm}) \\
(6)\end{array}$ & $\begin{array}{l}r_{+}^{\mathrm{aC}} \\
(\mathrm{nm}) \\
(7)\end{array}$ & $\begin{array}{l}r_{+}^{\mathrm{Si}} \\
(\mathrm{nm}) \\
(8)\end{array}$ \\
\hline HD149404 & 21 & $102 \pm 4$ & 12 & 8 & 3.0 & $101 \pm 23$ & $266 \pm 10$ & $348 \pm 13$ \\
\hline HD151804 & 20 & $88 \pm 2$ & 6 & 6 & 2.4 & $146 \pm 9$ & $358 \pm 11$ & $234 \pm 7$ \\
\hline HD152235 & 24 & $113 \pm 3$ & 15 & 7 & 2.7 & $101 \pm 17$ & $279 \pm 7$ & $194 \pm 5$ \\
\hline HD152248 & 19 & $74 \pm 2$ & 4 & 7 & 2.9 & $66 \pm 6$ & $419 \pm 12$ & $253 \pm 7$ \\
\hline HD152249 & 19 & $74 \pm 3$ & 9 & 6 & 3.0 & $218 \pm 9$ & $346 \pm 24$ & $263 \pm 18$ \\
\hline HD152408 & 21 & $79 \pm 2$ & 9 & 6 & 2.2 & $217 \pm 9$ & $335 \pm 9$ & $232 \pm 7$ \\
\hline HD153919 & 21 & $77 \pm 3$ & 12 & 6 & 2.8 & $104 \pm 2$ & $318 \pm 21$ & $324 \pm 21$ \\
\hline
\end{tabular}

NOTES - We convert specific to absolute dust abundances taking $15 \mathrm{ppm}$ of $[\mathrm{Si}] /[\mathrm{H}]$ locked in large $\left(6 \mathrm{~nm} \leq r \leq r_{+}^{\mathrm{Si}}\right)$ and the rest of $\mathrm{Si}$ in small $(r<6 \mathrm{~nm})$ silicates. This gives absolute abundances of (1) all Si, (2) all C, and of carbon in (3) graphite, and (4) PAH, respectively. Error of the abundance is $1 \sigma \sim 1 \mathrm{ppm}$ if not specified otherwise. (5) Exponent $q$ of the dust size distribution, $n(r) \propto r^{-q}$ (Mathis et al. [56]), $1 \sigma \sim 0.02$. (6) Minimum radius of aligned silicates $\left(r_{-}^{\mathrm{pol}}\right)$. Maximum radius of (7) silicates and (8) amorphous carbon grains.

line on a grey background that represents the $1 \sigma$ uncertainty. The circles show the photometry in the $U B V J H K$ bands. Model p8 is shown with a magenta line. For this model we show the contribution of each dust population to the extinction. The lower panel in each figure shows the linear polarisation spectrum $p(\lambda) / \tau_{V}$. The original (non rebinned) FORS spectra are represented with a grey line, while the circles represent the values rebinned to a spectral resolution $\Delta \lambda / \lambda \sim 50$. The best-fit (obtained with large aligned silicate grains) is represented with a magenta line.

We applied the conversion of specific to absolute dust abundances as described in Sect. 2. The total $\mathrm{Si}$ and $\mathrm{C}$ abundance in the dust model is denoted by $[\mathrm{Si}]^{\mathrm{tot}} /[\mathrm{H}]$ and $[\mathrm{C}]^{\mathrm{tot}} /[\mathrm{H}]$, respectively. They are given for each target in Table 3 together with absolute $\mathrm{C}$ abundance in graphite and PAHs and the other fitting parameters. 

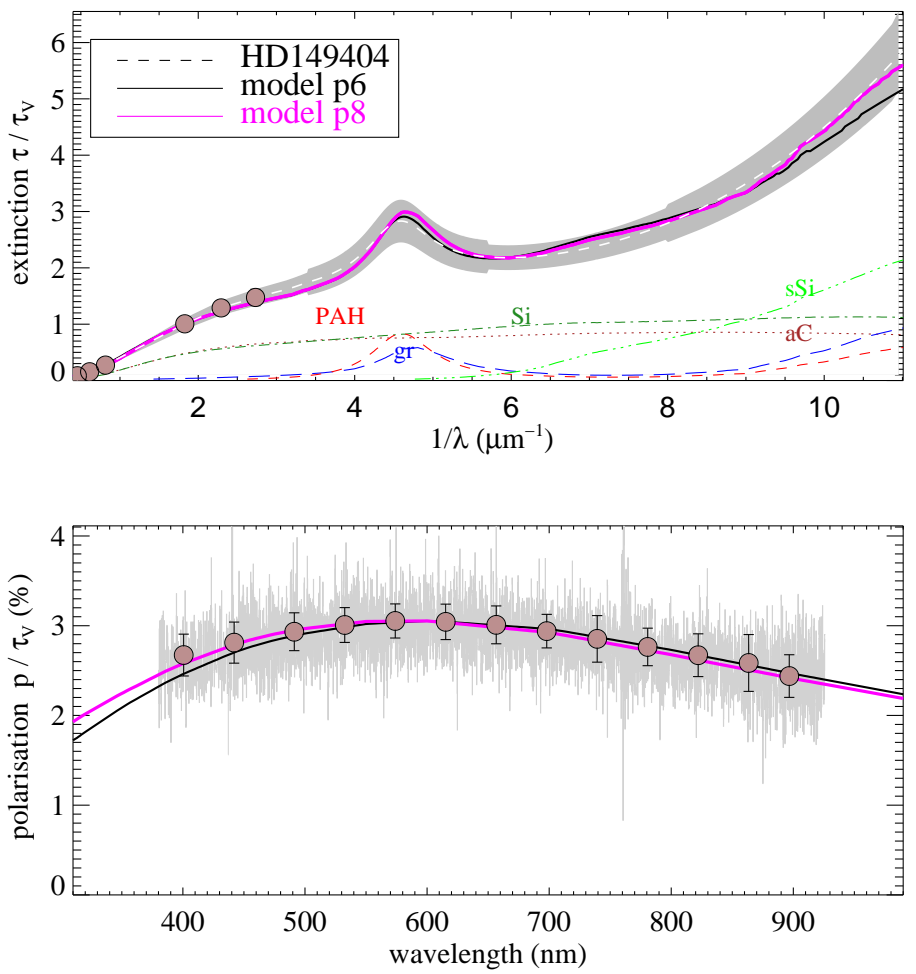

Figure 2: Fit to the extinction (Valencic et al. [72], Fitzpatrick [30]) and polarisation (this work) curve of HD 149404. Model p8 (magenta) and p6 (black) are shown by full lines. Top: Observed extinction (white dashed line) with $1 \sigma$ uncertainty (hashed area in grey), symbols refer to the UBVJHK photometry. The contribution of the different dust populations to the extinction is given for model $\mathrm{p} 8$ as labeled. Bottom: FORS polarisation spectrum (grey) and reduced to a spectral resolution of $\lambda / \Delta \lambda \sim 50$ (filled circles) with $1 \sigma$ error bars.

Cosmic element abundances are difficult to estimate and even more so is their depletion in dust. The sun's heavy elements abundances (Asplund et al. [6]) are higher than for B star that are in the solar vicinity. Therefore, we take as reference a gas phase carbon abundance $214 \pm 20 \mathrm{ppm}$ (Nieva \& Przybilla [60], Lyubimkov et al. [52], Snow \& Witt [69]). Nieva \& Przybilla [60] report a C abundance in ISM dust of $123 \pm 23 \mathrm{ppm}$ that can be reduced to $84 \pm 23 \mathrm{ppm}$ when the higher gas phase abundance by Sofia [70] is considered. Parvathi et al. [62] gives dust phase abundances of $\mathrm{C}$ for 11 sight-lines at $\mathrm{a} \geq 3 \sigma$ confidence level. We correct their values to the above reference and derive a 

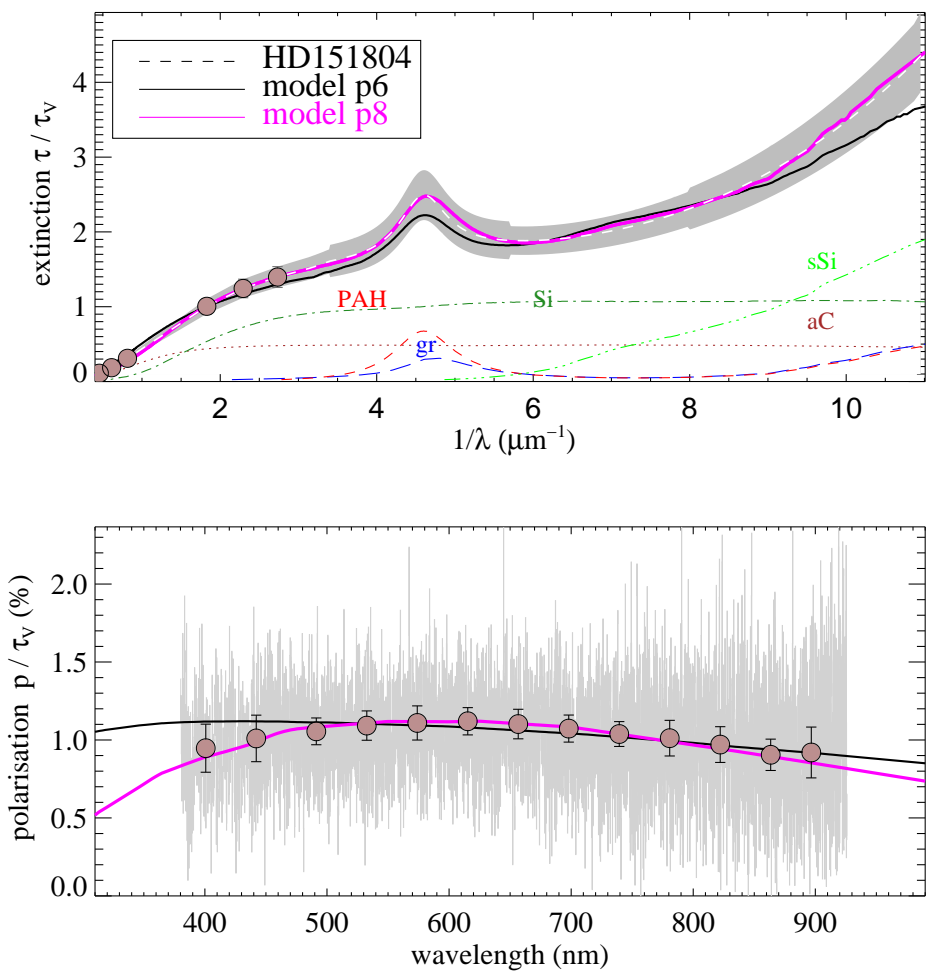

Figure 3: Same as Fig. 2 for HD 151804.

median $[\mathrm{C}] /[\mathrm{H}]_{\text {dust }}=102 \pm 47 \mathrm{ppm}$. Gerin et al. [36] finds a gas phase $\mathrm{C}$ abundance between $50-240 \mathrm{ppm}$. If one assumes that $50 \%$ of $\mathrm{C}$ is depleted into dust grains the $\mathrm{C}$ abundance in the dust is $75 \mathrm{ppm}$. The interstellar absorption feature at $3.4 \mu \mathrm{m}$ can be explained by hydrogenated amorphous carbon with a required number of carbon atoms in these grains of $72-97 \mathrm{ppm}$ derived by Duley et al. [25] and $80 \mathrm{ppm}$ by Furton et al [35]. In model $\mathrm{p} 8$ the median $\mathrm{C}$ abundance in dust is $79 \pm 14 \mathrm{ppm}$, which is in agreement with the previous estimates.

Nieva \& Przybilla [60] derive $[\mathrm{Si}] /[\mathrm{H}]_{\text {dust }}=29.4 \pm 3.6 \mathrm{ppm}$, whereas Voshchinnikov \& Henning [78] find, for similar sight-lines in the diffuse ISM as in our sample, $15 \leq[\mathrm{Si}] /[\mathrm{H}]_{\text {dust }} \leq 31.4(\mathrm{ppm})$ and a mean of $24 \pm 3 \mathrm{ppm}$. In model p8 the Si dust abundance range between $19-24 \mathrm{ppm}$ at a mean of $21 \pm 2 \mathrm{ppm}$. However, there are also open discussions on the $\mathrm{Fe}$ and $\mathrm{O}$ dust phase abundances (Dwek [27], Jenkins 

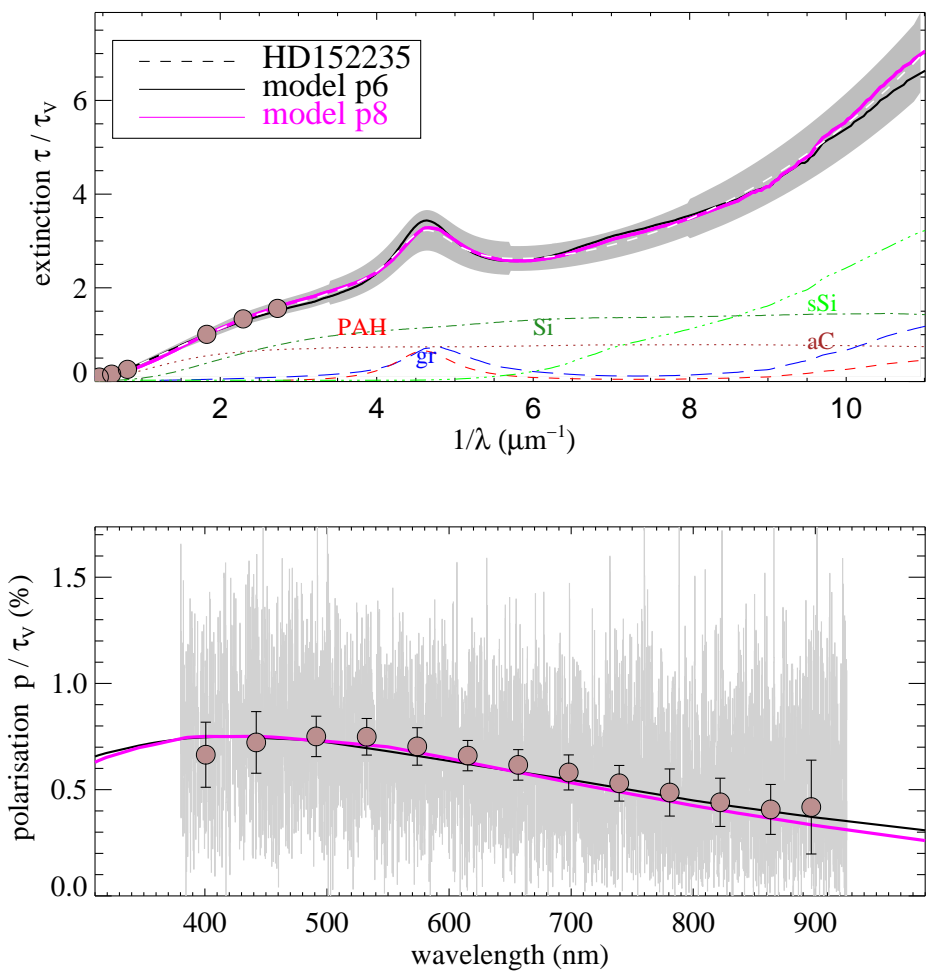

Figure 4: Same as Fig.2 for HD 152235.

[42]) and the detailed mineralogy of silicate grains (Henning [39]).

The polarisation curves are explained by large aligned silicates with average minimum alignment radii of $\left\langle r_{-}^{\mathrm{pol}}\right\rangle=140 \pm 56(\mathrm{~nm})$ and maximum radius $\left.<r_{+}^{\mathrm{Si}}\right\rangle=$ $264 \pm 50(\mathrm{~nm})$, respectively. For HD 152248 and HD 152249 the observed polarisation is below $0.5 \%$. The upper radius of carbon grains is $\sim 335 \pm 50 \mathrm{~nm}$, so about $25 \%$ larger than for silicate grains (Table 3). Probably, this is because the efficiency of destruction of carbonaceous particles is lower (Slavin et al. [68]). Note that Hirashita \& Voshchinnikov [40] presented a dust model fitting extinction data by grain growth. They need to tune the accretion and coagulation process and increase the sticking coefficient of silicates so that silicates become larger than carbon grains.

We have extensively explored whether other dust models with a smaller number of free parameters could satisfy extinction, polarisation and abundance constraints. As a 

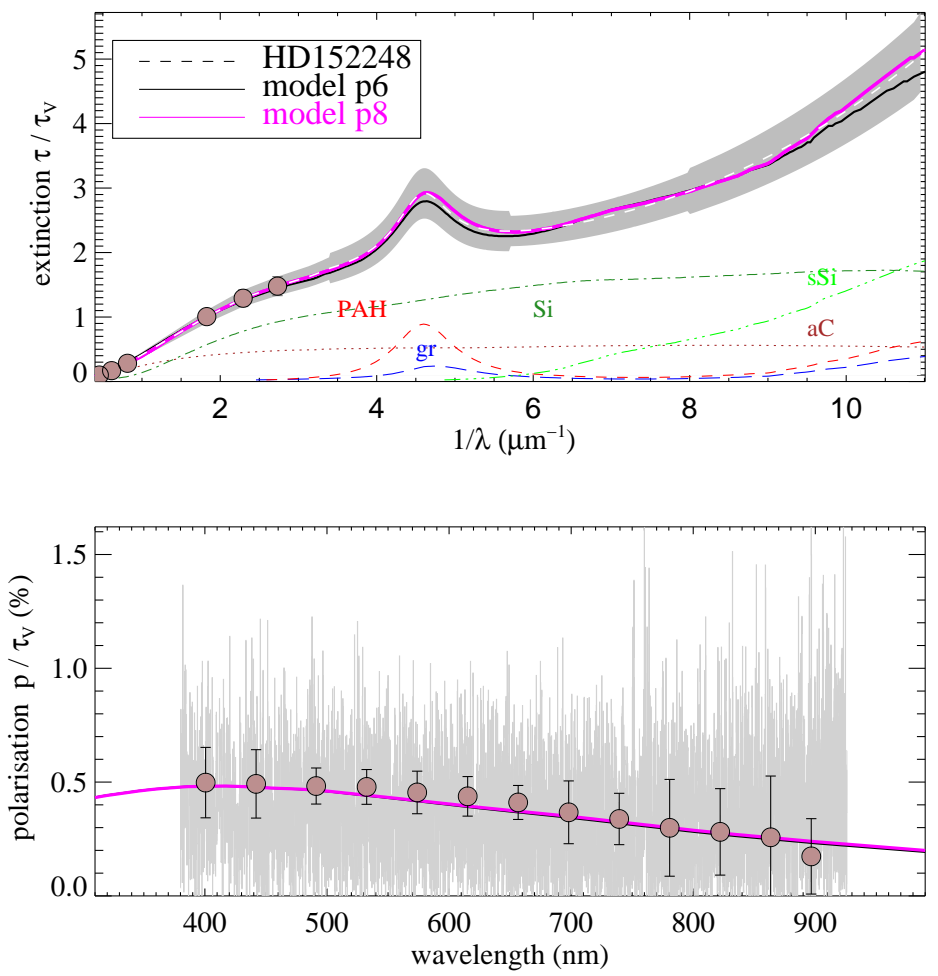

Figure 5: Same as Fig.2 for HD 152248.

first attempt, we simplified our p8 model by assuming that the upper radius of large silicates and carbon dust are identical $\left(r_{+}^{\mathrm{Si}}=r_{+}^{\mathrm{aC}}\right)$. The best-fits to the extinction and polarisation curve of such a model are good. However, it requires more $\mathrm{C}$ than model p8.

We considered also a model with six free parameters (that we will call p6), by fixing $q=3.1$, assuming that the $\mathrm{C}$ abundance in PAHs is $8 \%$ the $\mathrm{C}$ abundance in large carbon grains $\left(\Upsilon_{\mathrm{PAH}}=8 \% \Upsilon_{\mathrm{aC}}\right)$, and keeping as free parameters the quantities $\Upsilon_{\mathrm{aC}}, \Upsilon_{\mathrm{gr}}$, $\Upsilon_{\mathrm{sSi}}, r_{-}^{\mathrm{pol}}, r_{+}^{\mathrm{Si}}$, and $r_{+}^{\mathrm{aC}}$. It is shown as black lines in Figs. 2-8. Model p6 provides good fits to the extinction and polarisation spectra, however it produces somewhat larger deviations from the data than model $\mathrm{p} 8$. This is evident inspecting residuals of fits to the extinction, $\left(\tau^{\text {mod }}-\tau^{\text {data }}\right) / \tau^{\text {data }}$, and polarisation, $\left(p^{\text {mod }}-p^{\text {data }}\right) / p^{\text {data }}$. They are displayed in Fig. 9 . For some stars model p6 has difficulties accounting for the steep 

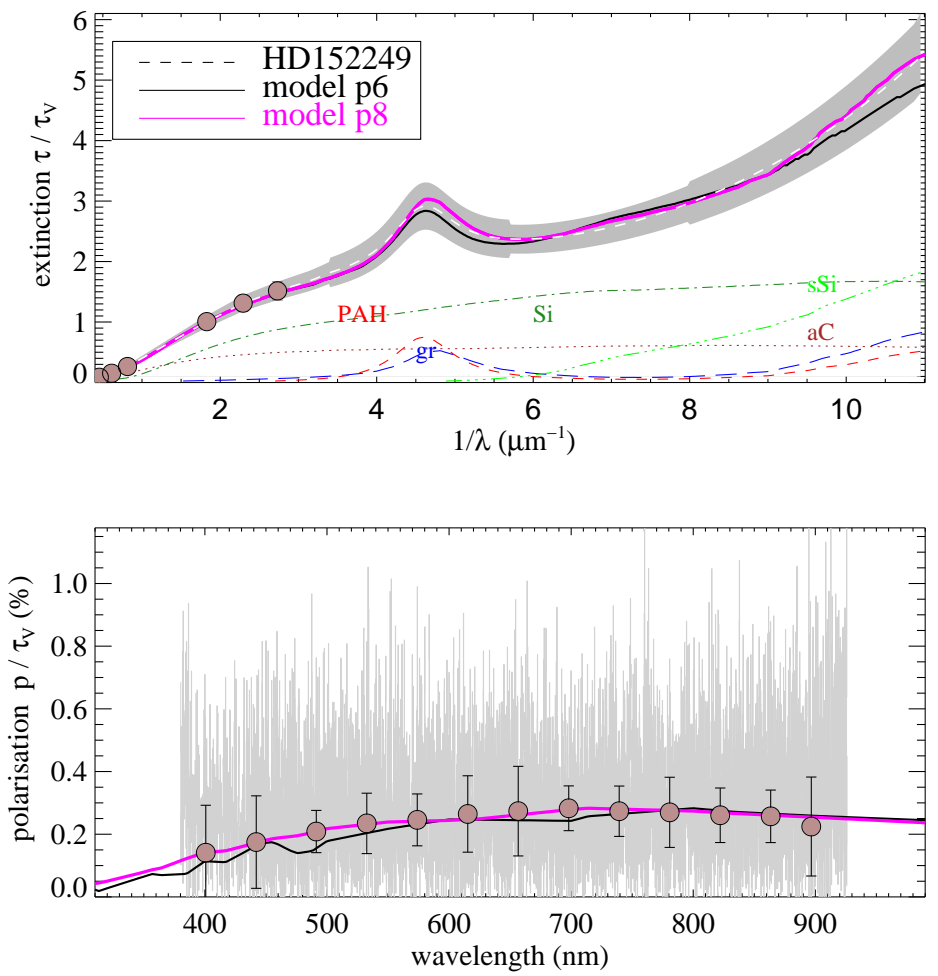

Figure 6: Same as Fig.2 for HD 152249.

rise in the far UV (at $x \gtrsim 10 \mu \mathrm{m}^{-1}$ ), and for two stars it underestimates the extinction bump, and overestimates the near IR extinction. Model p6 accounts for the $[\mathrm{Si}]^{\text {tot }} /[\mathrm{H}]$ abundance constraints but requires $50 \%$ more $\mathrm{C}$ than model $\mathrm{p} 8$.

Finally, we apply a model where we include contributions by large grains and PAHs and assume $r_{+}^{\mathrm{Si}}=r_{+}^{\mathrm{aC}}$. This model ignores very small silicates and graphite grains. It reproduces the polarisation spectra. The model fits the extinction curve for $x \lesssim$ $7 \mu \mathrm{m}^{-1}$. However, at shorter wavelength it stays rather flat and fails to account for the steep extinction rise in the far UV. This model accounts for the $[\mathrm{Si}]^{\text {tot }} /[\mathrm{H}]$ abundance constraints and requires $30 \%$ more $\mathrm{C}$ than model $\mathrm{p} 8$. 

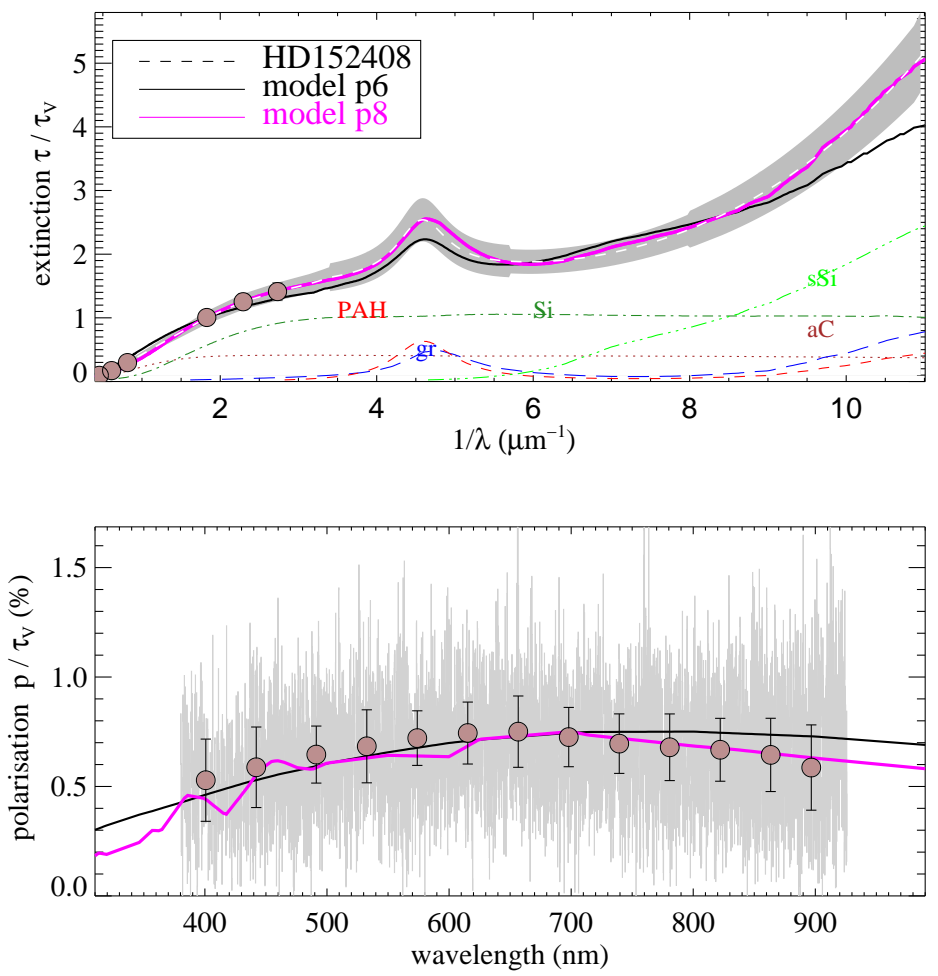

Figure 7: Same as Fig.2 for HD 152408.

\section{Conclusion}

We have presented some preliminary results of our Large Interstellar Polarisation Survey (LIPS) at the VLT, using a selected sub-sample of seven stars along lines-ofsight towards the Sco OB1 association. For these stars we have obtained high S/N polarisation spectra with the FORS2 instrument in the spectral range $365-920 \mathrm{~nm}$, while extinction curves were taken from the literature [72]. The mean $R_{V} \sim 3.7$ of our sample is somewhat larger than the typical value of $R_{V} \sim 3.1$ of the diffuse ISM (Fitzpatrick \& Massa [33]).

We explained the dust extinction and polarisation spectra with a model of the interstellar dust that includes a population of carbon and silicate grains with a power-law size distribution ranging from the molecular domain $(0.5 \mathrm{~nm}$ and PAHs) up to $800 \mathrm{~nm}$. 

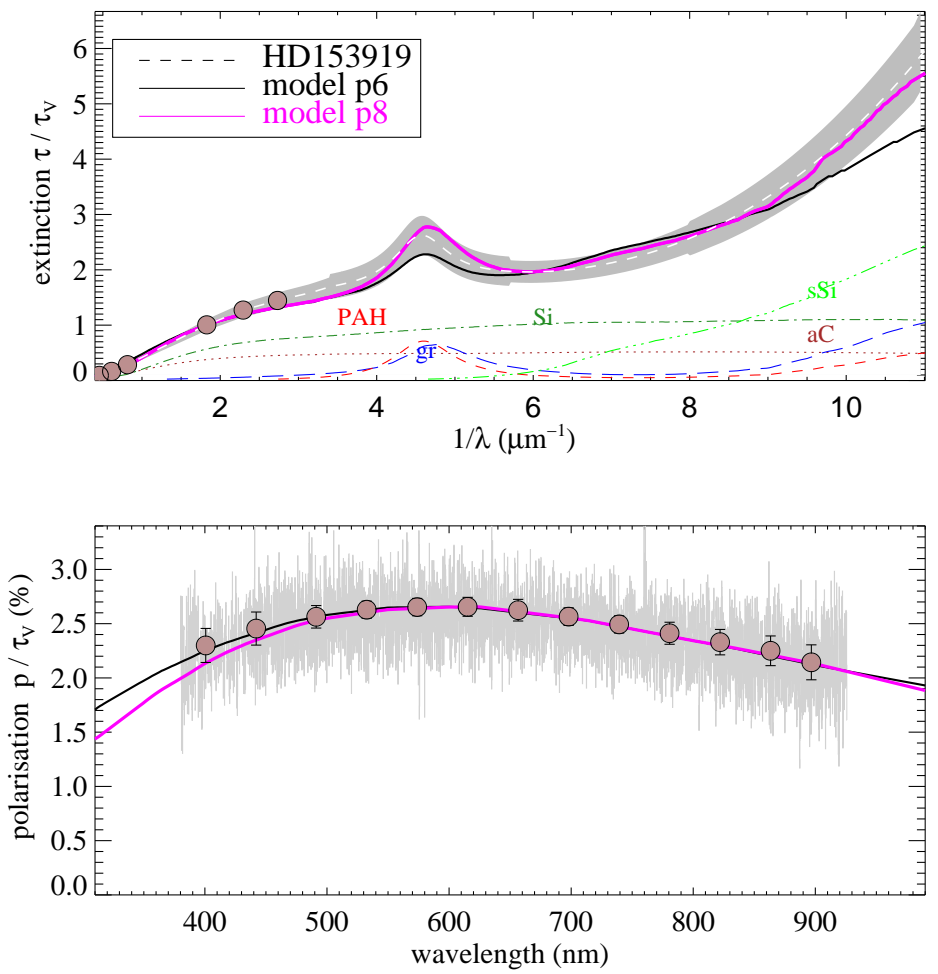

Figure 8: Same as Fig.2 2 for HD 153919

Particles with radii larger than $6 \mathrm{~nm}$ are spheroids. Dust cross section computed in the optical/UV with different sets of optical constants (Zubko et al. [85], Draine [17], Jones [44], Jones et al. [45]) are similar.

Extinction and polarisation data are best-fit by assuming dust models with different numbers of free parameters. We vary the exponent of the size distribution $q$, specific abundances of $\mathrm{C}$ in amorphous carbon $\Upsilon_{\mathrm{aC}}$, graphite $\Upsilon_{\mathrm{gr}}$, and PAHs $\Upsilon_{\mathrm{PAH}}$, as well as $\mathrm{Si}$ in small silicates $\Upsilon_{\mathrm{SSi}}$, the minimum radius of aligned silicates $r_{-}^{\mathrm{pol}}$, and the upper grain radius of silicates $r_{+}^{\mathrm{Si}}$ and amorphous carbon $r_{+}^{\mathrm{aC}}$. A model where all eight parameters are varied seems to be in reasonable agreement with present abundance constraints of $\mathrm{C}$ and $\mathrm{Si}$ in dust. Equally good fits are found assuming the same upper grain radius for both dust components. However, such a model overestimates the $\mathrm{C}$ abundance in two out of seven sight lines studied. We find another model with less (6) free parameters 

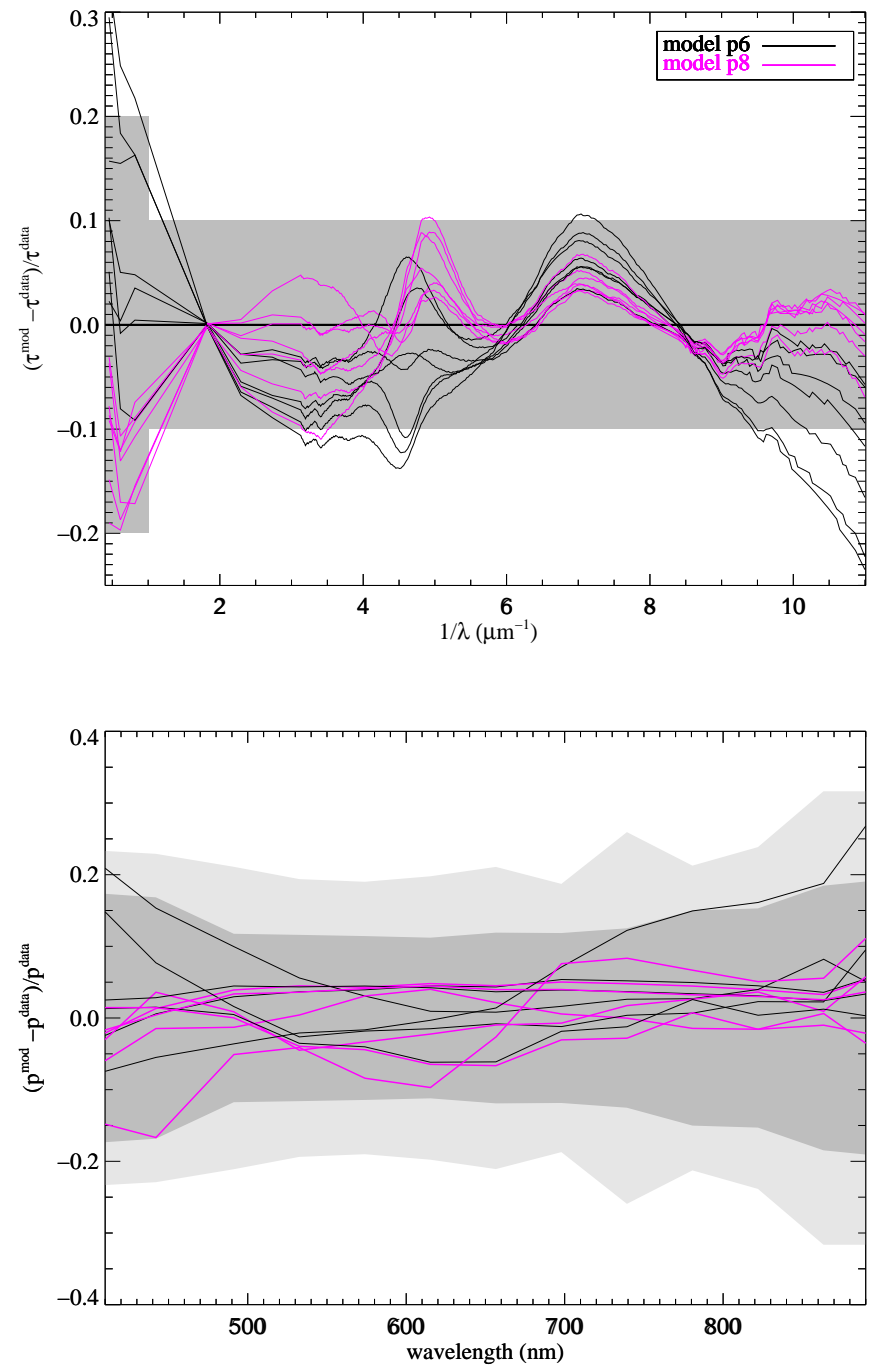

Figure 9: Residuals of model p8 (magenta) and model p6 (black) to the extinction (top) and polarisation data (bottom) presented for targets in Fig. 2- Fig. 8 The hashed area in grey indicates a typical $1 \sigma$ error and the light grey area in the bottom panel mark the maximum $1 \sigma$ error in the binned polarisation spectra.

that fit data down to the lowest IUE observing range $\left(x \lesssim 8.6 \mu \mathrm{m}^{-1}\right)$. However, it overestimates the $\mathrm{C}$ abundance in dust by $\sim 50 \%$. We analysed another model that includes large grains and a PAH component but ignores small graphite and small silicates. We 
keep in that model $r_{+}^{\mathrm{Si}}=r_{+}^{\mathrm{aC}}$. Such a model provides fits at somewhat larger reduced $\chi^{2}$, requires $\sim 30 \%$ more carbon than model $\mathrm{p} 8$, and fails accounting for the steep rise of the extinction curve in the far UV at $x \gtrsim 7 \mu \mathrm{m}^{-1}$. We take it as a demonstration that beside PAHs, dust populations of very small silicate and graphite grains are needed.

We conclude that data are best fit by a model with eight free parameters. From our modelling of the sight lines we found that the exponent of the dust size distribution is $q \sim 2.7 \pm 0.3$. The upper size of large silicates ranges between 194 and $348 \mathrm{~nm}$, and that of amorphous carbon grains is $\sim 25 \%$ larger. This is in agreement with recent estimates of grain destruction efficiencies (Slavin et al. [68]). We have converted the specific abundances of the model into absolute abundances by assuming that $15 \mathrm{ppm}$ of $\mathrm{Si}$ is locked in large silicate grains. Our model requires $6 \pm 2 \mathrm{ppm}$ of $\mathrm{Si}$ in very small silicates. The abundance of $\mathrm{C}$ in graphite compared to the total $\mathrm{C}$ in dust is $\Upsilon_{\mathrm{gr}} / \Upsilon_{\text {tot }}=11 \pm 3(\%)$ and that of PAH $\Upsilon_{\mathrm{PAH}} / \Upsilon_{\text {tot }}=8 \pm 1(\%)$. The polarisation curves are explained by large aligned silicates with minimum alignment radii $66 \leq r_{-}^{\text {pol }} \leq 218(\mathrm{~nm})$. Typical numbers of the individual dust abundances and other parameters derived from the complete LIPS sample will be presented in a forthcoming paper.

\section{Acknowledgments}

The anonymous referees are thanked for their careful read and helpful suggestions improving the quality of the article. In particular Referee 2 is warmly thanked for drawing our attention to cosmic abundance constraints in the gas phase and solids of the ISM, and also the papers discussing the bulk density of amorphous carbon. We thank Endrik Krgel for various discussions. NVV was partly supported by the RFBR grant 16-02-00194. This work is based on observations made with ESO Telescopes at the La Silla Paranal Observatory under programme ID 095.C-0855(A) and 096.C0159(A). This research has made use of the SIMBAD database, operated at the CDS, Strasbourg, France. 


\section{References}

[1] Anderson, C.M., Weitenback, A.J., Code, A.D., Nordsieck, K.H., Meade, M.R., et al. 1996, AJ, 112, 2726

[2] Andersson B. - G., Lazarian A. \& Vaillancourt J. E. 2015, ARAA, 53, 501

[3] Ankay, A., Guseinov, O. H., Alpar, M. A., Tagieva, S. O. 2001, astro$\mathrm{ph} / 0102118 \mathrm{v} 1$

[4] Appenzeller, I., Fricke, K., Furtig, W., et al. 1998, The Messenger, 94, 1

[5] Asano, Ryosuke S., Takeuchi, Tsutomu T., Hirashita, Hiroyuki, Nozawa, Takaya 2014, MNRAS, 440, 134

[6] Asplund, M., Grevesse, N., Sauval, A.J., \& Scott, P. 2009 ARA\&A, 47, 481

[7] Bagnulo, S., Landolfi, M., Landstreet, J.D., et al. 2009, PASP, 121, 993

[8] Bless, R. C., Savage, B. D. 1970, IAUS, 36, 38

[9] Bruzual A., G., Magris, G., \& Calvet, N. 1988, ApJ, 333, 673

[10] Calzetti, D., Kinney, A. L., \& Storchi-Bergmann, T. 1994, ApJ, 429, 582

[11] Cardelli, Jason A., Savage, Blair D. 1988, ApJ, 325, 864

[12] Casiraghi, C., Ferrari, A. C., and Robertson, J. 2005, Physical Review B 72.8

[13] Clayton, G. C., Mathis, J. S., Cardelli, J. A. 1986, BAAS, 18, 1028

[14] Das, H.K., Voshchinnikov, N.V., \& Ilín, V.B. 2010, MNRAS 404, 625

[15] Désert, F.X., Boulanger, F., \& Puget, J.L. 1990, A\&A, 237, 215

[16] Dobashi, Kazuhito, Uehara, Hayato, Kandori, et al. 2005, PASJ, 57, 1

[17] Draine, B.T. 2003, ApJ, 598, 1026

[18] Draine, B.T. 2011, Physics of the Interstellar and Intergalactic Medium, Princton University 
[19] Draine, B. T. 2009, ASPC, 414, 453

[20] Draine, B.T., \& Fraisse, A.A. 2009, ApJ, 626, 1

[21] Draine, B.T., \& Hensley, B. 2013, ApJ, 765, 159

[22] Draine, B. T., Hensley, Brandon S. 2016, arXiv160506671

[23] Draine, B.T., \& Li, A. 2007, ApJ, 657, 810

[24] Draine, B.T., \& Malhotra, S. 1993, ApJ, 414, 632

[25] Duley, W. W., Scott, A. D., Seahra, S., and Dadswell, G. 1998, ApJ 503, L183

[26] Dwek, E., Arendt, R.G., Fixsen, D.J., Sodroski, T.J., Odegard, N., et al. 1997, ApJ, 475, 565

[27] Dwek, E., 2016, ApJ, 825, 136

[28] Fischera, J. \& Dopita, M. 2005, ApJ, 619, 340

[29] Fitzpatrick, E.L. 1999, PASP, 111, 163

[30] Fitzpatrick, E. L. 2004, ASPC, 309, 33

[31] Fitzpatrick, E. L. \& Massa, D. 1986, ApJ, 307, 286

[32] Fitzpatrick, E. L. \& Massa, D. 1990, ApJS, 72, 163

[33] Fitzpatrick, E. L. \& Massa, D. 2007, ApJ, 663, 320

[34] Furton, D. G., and Witt, A.N. 1993 ApJ 415, L51

[35] Furton D. G., Laiho, J.W., and Witt, A. N. 1999, ApJ, 526, 752

[36] Gerin, M., Ruaud, M., Goicoechea, J. R., Gusdorf, A., Godard, B. et al. 2015, A\&A, 573, 30

[37] Goodman, Alyssa A., Jones, Terry J., Lada, Elizabeth A., Myers, Philip C. 1995, ApJ, 448, 748

[38] Gordon, K.D., Cartledge, S., \& Clayton, G.C. 2009, ApJ, 705, 1320 
[39] Henning, Th. 2010, ARA\&A, 48, 21

[40] Hirashita, Hiroyuki \& Voshchinnikov, Nikolai V. 2014, MNRAS, 437, 1636

[41] Indebetouw, R., Whitney, B. A., Johnson, K. E., Wood, K. 2006, ApJ, 636, 362

[42] Jenkins, E.B. 2009, ApJ, 700, 1299

[43] Johnson, H.L. 1968, Nebulae and interstellar matter. Edited by Barbara M. Middlehurst; Lawrence H. Aller. Library of Congress Catalog Card Number 66-13879. Published by the University of Chicago Press, Chicago, ILL USA, 1968, p.167

[44] Jones, A. P. 2012 A\&A, 540, A2 (corrigendum 2012, A\&A, 545, C2)

[45] Jones, A. P., Fanciullo, L., Köhler, M., et al. 2013, A\&A, 558, 62

[46] Kim, S.H., Martin, P.G., \& Hendry, P.D. 1994, ApJ, 422, 164

[47] Köhler, M., Jones, A., Ysard, N. 2014, A\&A, 565, L9

[48] Krügel, E. 2008, An introduction to the Physics of Interstellar Dust, IoP, ISBN 9781

[49] Krügel, E. 2009, A\&A, 493, 385

[50] Lallement, R., Vergely, J.-L., Puspitarini, L., et al., 2015, MmSAI, 86, 626

[51] Lazarian, A. 2003, JQSRT, 79, 881

[52] Lyubimkov, Leonid S.; Lambert, David L.; Poklad, Dmitry B.; Rachkovskaya, Tamara M.; Rostopchin, Sergey I. 2013, MNRAS, 428, 3497

[53] Markwardt, C. B. 2009, ASPC, 411, 251

[54] Mathis, J.S. 1990, ARA\&A, 28, 37

[55] Massa, D., Savage, B. D., Fitzpatrick, E. L. 1983, ApJ, 266, 662

[56] Mathis, J.S., Rumpl, W., \& Nordsieck, K.H. 1977, ApJ, 217, 425 
[57] More, J. 1977, The Levenberg-Marquardt Algorithm: Implemen tation and Theory , in Numerical Analysis, vol. 630, ed. G. A. Watson (Springer-V erlag: Berlin), 105

[58] More, J. \& Wright, S. 1993, Optimization Software Guide, Frontiers in Applied Mathematics, vol. 14, (Philadelphia, PA: SIAM)

[59] Natta, A., \& Panagia, N. 1984, ApJ, 287, 228

[60] Nieva, M.-F., Przybilla, N. 2012, A\&A, 539, 143

[61] Papaj, J., Krelowski, J. 1992, AcA, 42, 211

[62] Parvathi, V.S., Sofia, U.J., Murthy, J., \& Babu, S.R.S. 2012, ApJ, 760, 36

[63] Robertson, J. 2002, Mat. Sci. Eng. R 37, 129

[64] Scicluna, P., Siebenmorgen, R. 2015, A\&A, 584, A108

[65] Serkowski, K., Mathewson, D.S., \& Ford, V.L. 1975, ApJ, 196, 261

[66] Siebenmorgen, R., Voshchinnikov, N. V., Bagnulo, S. 2014, A\&A, 561, A82

[67] Siebenmorgen, R., \& Krügel, E. 1992, A\&A, 259, 614

[68] Slavin, J. D., Dwek, E., \& Jones, A. P. 2015, ApJ, 803, 7

[69] Snow, Theodore P., Witt, Adolf N. 1996, ApJ, 468, 65

[70] Sofia, U., J. 2004, ASPC, 309, 393

[71] van Leeuwen, F. 2007, A\&A, 474, 653

[72] Valencic, Lynne A., Clayton, Geoffrey C., Gordon, Karl D. 2004, ApJ, 616, 912

[73] Voshchinnikov, N. V. 2012, JQSRT, 113, 2334

[74] Voshchinnikov, N. V., Molster, F. J., The, P. S. 1995, Ap\&SS, 224, 223

[75] Voshchinnikov, N.V., Henning, Th., Prokopjeva, M.S., \& Das, H.K. 2012, A\&A, 541, A52 
[76] Voshchinnikov, N. V., Il'in, V. B., Das, H. K. 2016, MNRAS, 462, 2343

[77] Voshchinnikov, N.V. \& Farafonov, V.G. 1993, Ap\&SS 204, 19

[78] Voshchinnikov, Nikolai V. \& Henning, Th. 2010, A\&A, 517, A45

[79] Voshchinnikov, Nikolai V., \& Hirashita, Hiroyuki 2014, MNRAS, 445, 301

[80] Weingartner, J.C., \& Draine, B.T. 2001, ApJ, 548, 296

[81] Whittet, D.C.B. 2003, in Dust in the Galactic Environment, Bristol: Institute of Physics Publishing

[82] Witt, A. N. \& Gordon, K. D. 1996, ApJ, 463, 681

[83] Witt, A. N. \& Gordon, K. D. 2000, ApJ, 528, 799

[84] Wolf, S., Fischer, O., \& Pfau, W. 1998, A\&A, 340, 103

[85] Zubko, V.G., Mennella, V., Colangeli, L., et al. 1996, MNRAS, 282, 1321 